\title{
Assessment of Rashba Field Effects in Ultrathin Pt/Co/GdOx Submicrometer Strips
}

\author{
Satoru Emori and Geoffrey S. D. Beach \\ Department of Materials Science and Engineering, Massachusetts Institute of Technology, Cambridge, MA 02139 USA
}

\begin{abstract}
The influence of injected current on magnetization dynamics is investigated in submicrometer-wide strips of out-of-plane magnetized $\mathrm{Pt} / \mathrm{Co} / \mathrm{GdOx}$. This ultrathin film structure is similar to $\mathrm{Pt} / \mathrm{Co} / \mathrm{AlOx}$ exhibiting highly efficient current-driven domain wall (DW) motion, which has recently been attributed in part to the Rashba effect. In Pt/Co/GdOx, the reduction of the out-of-plane magnetization with increasing injected current is largely due to Joule heating, and the effective transverse Rashba field that tilts the magnetization is estimated to be at most $\sim 0.3 \mathrm{~T} /\left(10^{12} \mathrm{~A} / \mathrm{m}^{2}\right)$. Measurements of high-speed DW motion reveal no evidence of a strong Rashba field to suppress DW precession. These findings indicate that the Rashba effect plays a limited or negligible role in current-driven DW dynamics, and alternative torques are required to explain the high efficiency of DW motion in ultrathin heavy-metal/ferromagnet/oxide systems.
\end{abstract}

Index Terms-Magnetic domain walls, magnetic multilayers, magnetoelectronics, perpendicular magnetic anisotropy.

\section{INTRODUCTION}

C ONTROL OF magnetic domain walls (DWs) by electric current in thin-film-based patterned devices is crucial for developing low-energy, high-speed, and scalable nonvolatile magnetic memory and logic devices. Out-of-plane magnetized thin films with strong perpendicular magnetic anisotropy are promising platforms for such devices, because of the narrow widths and lower threshold current densities for motion of DWs in these material systems. Recently, highly efficient currentdriven DW motion has been observed in out-of-plane magnetized trilayer structures consisting of an ultrathin $(<1 \mathrm{~nm})$ ferromagnetic Co layer embedded between a heavy-metal Pt underlayer and an oxide overlayer such as AlOx [1] or GdOx [2], with maximum DW velocities exceeding $100 \mathrm{~m} / \mathrm{s}$. The conventional adiabatic and nonadiabatic spin transfer torques (STTs) [3] cannot account for the high efficiencies or the current polarity-dependence of DW motion, which occurs in the direction opposing electron flow, in these $\mathrm{Pt} / \mathrm{Co} /$ oxide strips. Moreover, because the spin polarization of in-plane injected current may be small in such a thin ferromagnetic layer [4], conventional STTs are expected to be weak. Instead, current-induced torques arising from interfacial effects [5]-[7] are likely responsible for DW motion.

One interfacial effect that may contribute to highly efficient current-induced DW motion in $\mathrm{Pt} / \mathrm{Co} /$ oxide is the Rashba effect [8]. In an asymmetric out-of-plane electric potential profile (i.e., one side bounded by a heavy metal and the other by an oxide), conduction electron spins are canted in an effective Rashba magnetic field $H_{R}$ arising from spin-orbit coupling, and these conduction spins exert a torque on the magnetization within the ultrathin magnetic layer. The direction of $H_{R}$ is in-plane and transverse to the strip (Fig. 1(a)), and its magnitude was reported to reach $\mu_{0}\left|H_{R}\right| \approx 1 \mathrm{~T}$ per $10^{12} \mathrm{~A} / \mathrm{m}^{2}$ of injected current [8]. According to Miron et al. [1], this large

Manuscript received November 05, 2012; revised February 09, 2013; accepted February 27, 2013. Date of current version July 15, 2013. Corresponding author: S. Emori (e-mail: satorue@mit.edu).

Color versions of one or more of the figures in this paper are available online at http://ieeexplore.ieee.org.

Digital Object Identifier 10.1109/TMAG.2013.2251459 (a)

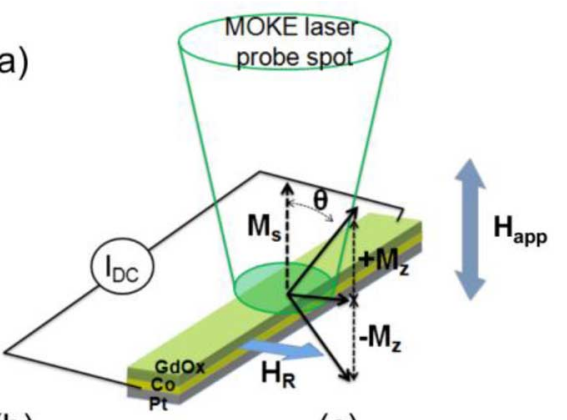

(b)

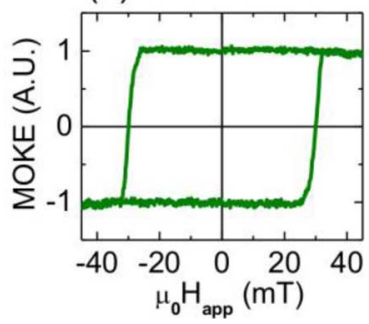

(c)

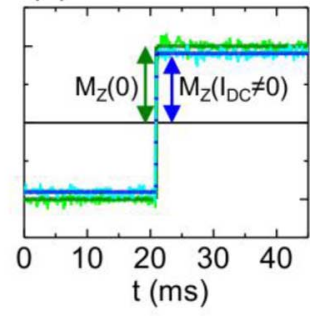

Fig. 1. (a) Schematic of magnetization tilting measurement. The out-of-plane magnetization $M_{\mathrm{Z}}$ is measured using the magneto-optical Kerr effect (MOKE). The injected DC current $I_{\mathrm{DC}}$ may generate a transverse Rashba field $H_{\mathrm{R}}$, which may tilt the magnetization and reduce $M_{\mathrm{Z}}$. The out-of-plane applied field $H_{\text {app }}$ switches $M_{\mathrm{Z}}$ between up and down. (b) MOKE hysteresis loop measured at $I_{\mathrm{DC}}=0$ by sweeping out-of-plane $H_{\mathrm{app}}$. (c) Examples of MOKE signal upon magnetization switching for zero and nonzero $I_{\mathrm{DC}}$. The magnitude of the MOKE signal is proportional to $M_{\mathrm{Z}}$

$H_{R}$ could lock the configuration of moving DWs and allow high-speed DW motion by preventing turbulent processional motion (Walker breakdown) that limits the velocity of DWs propelled by large driving forces. The existence of the Rashba field has been confirmed independently in $\mathrm{Pt} / \mathrm{Co} / \mathrm{AlOx}$ [9] and $\mathrm{Ta} / \mathrm{CoFeB} / \mathrm{MgO}$ [10], although the magnitudes reported by these studies are smaller at $\sim 0.3 \mathrm{~T} /\left(10^{12} \mathrm{~A} / \mathrm{m}^{2}\right)$. Another study on $\mathrm{Pt} / \mathrm{Co} / \mathrm{AlOx}$ has reported a negligible Rashba field of $<0.013 \mathrm{~T} /\left(10^{12} \mathrm{~A} / \mathrm{m}^{2}\right)$ [11].

If current-driven DW motion is indeed facilitated by the Rashba effect, further enhancement may be expected in material systems with stronger spin-orbit coupling at the $\mathrm{Co} /$ oxide interface. Large Rashba splitting has previously been observed at the surface of oxidized Gd [12], suggesting that strong 
Rashba spin-orbit coupling may also arise at the $\mathrm{Co} / \mathrm{GdOx}$ interface. Our recent studies on ultrathin $\mathrm{Pt} / \mathrm{Co} / \mathrm{GdOx}$ films have demonstrated that an electric field applied across the GdOx layer can modify magnetic anisotropy (thereby controlling DW motion) [13], [14], implying that significant spin-orbit coupling exists at the $\mathrm{Co} / \mathrm{GdOx}$ interface. Therefore, a Rashba effective field comparable to or even in excess of that reported in $\mathrm{Pt} / \mathrm{Co} / \mathrm{AlOx}$ [8] may be expected in $\mathrm{Pt} / \mathrm{Co} / \mathrm{GdOx}$.

In this report, we examine the role of the current-induced Rashba effect on DW motion in submicrometer-wide strips of $\mathrm{Pt} / \mathrm{Co} / \mathrm{GdOx}$. We determine an upper limit on the transverse Rashba field by measuring the current-induced tilting of the magnetization away from the out-of-plane easy axis. Our data show that the out-of-plane component of the magnetization vector decreases with increasing injected current density, but that the reduction can be fully attributed to the decrease in $M_{\mathrm{S}}$ due to Joule heating. The uncertainty of this measurement yields an upper limit $\mu_{0}\left|H_{R}\right|<0.3 \mathrm{~T} /\left(10^{12} \mathrm{~A} / \mathrm{m}^{2}\right)$, significantly lower than reported in [8]. We also analyze DW motion driven by field and current [2] and find no evidence of the Rashba effect suppressing the precessional mode of motion.

\section{EXPERIMENTAL METHODS}

The stack structure of the ultrathin film was $\mathrm{Si}$-substrate $/ \mathrm{SiO}_{2}(50) / \mathrm{Ta}(4) / \mathrm{Pt}(3) / \mathrm{Co}(0.9) / \mathrm{GdOx}(3)$, where the numbers in parentheses are layer thicknesses in $\mathrm{nm}$. The metal layers were deposited by DC magnetron sputtering under 3 mTorr of Ar at a background pressure of $\sim 1 \times 10^{-7}$ Torr. The GdOx films were grown by dc reactive sputtering of a metal Gd target in an oxygen partial pressure of $\sim 5 \times 10^{-5}$ Torr. The Ta underlayer enhanced perpendicular magnetic anisotropy in $\mathrm{Pt} / \mathrm{Co} / \mathrm{GdOx}$ and adhesion between the substrate and $\mathrm{Pt}$. Using vibrating sample magnetometry, the hard-axis (in-plane) saturation field was determined to be $\mu_{0} H_{\mathrm{K}} \sim 0.8 \mathrm{~T}$. The room-temperature saturation magnetization was close to the bulk value at $\sim 1.3 \times 10^{6} \mathrm{~A} / \mathrm{m}$, indicating minimal oxidation of the Co layer. Patterned 500-nm wide strip devices with $\mathrm{Ta}(3) / \mathrm{Cu}(100)$ electrodes were fabricated with electron beam lithography and liftoff.

Out-of-plane magnetization was measured with a high-bandwidth scanning polar magneto-optical Kerr effect (MOKE) polarimeter [15], with a focused beam spot size of $\sim 3 \mu \mathrm{m}$ positioned with a high-resolution sample scanning stage. An electromagnet was used to apply an out-of-plane field to saturate the magnetization or propagate DWs. The substrate temperature $T_{\text {sub }}$ was maintained at $308 \pm 0.1 \mathrm{~K}$ using a thermoelectric module installed directly on top of the electromagnet. The sample substrate and the thermocouple that measured $T_{\text {sub }}$ were both on the thermoelectric module. The procedures for the measurements of magnetization tilting and DW motion are described in the following respective sections.

\section{Current-Induced Magnetization Tilting}

In the thin-film strip geometry of Fig. 1(a), current injected along the strip axis is expected to generate a transverse Rashba field $H_{\mathrm{R}}$, which would cant the strip magnetization away from (a)

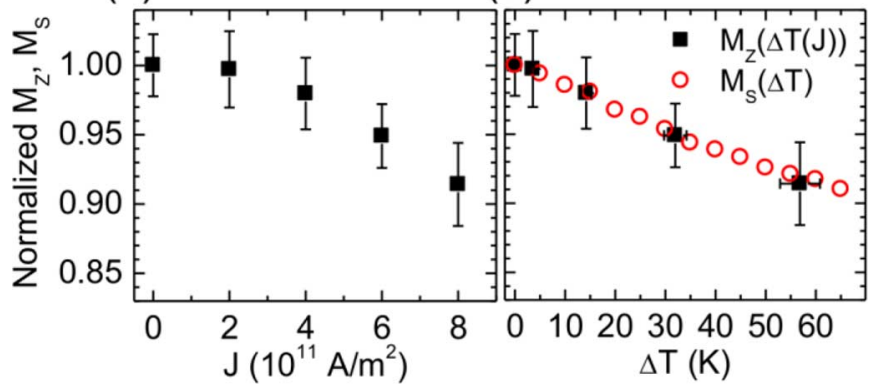

Fig. 2. (a) Decrease of the out-of-plane magnetization $M_{\mathrm{Z}}$ with increasing injected current density $J$ in the patterned device. (b) Decrease of $M_{Z}$ with increasing sample temperature resulting from Joule heating at a constant substrate temperature $(308 \mathrm{~K})$ in the submicrometer strip $\left(M_{\mathrm{Z}}(\Delta T(J))\right.$, filled squares), and decrease of $M_{\mathrm{S}}$ from increasing substrate temperature in the continuous film $\left(M_{\mathrm{S}}(\Delta T)\right.$, open circles $)$.

the easy axis. The tilting of magnetization as a function of injected DC current was characterized by measuring the MOKE signal at the center of a $\mathrm{Pt} / \mathrm{Co} / \mathrm{GdOx}$ strip, which is proportional to the out-of-plane component $M_{\mathrm{Z}}$ of the magnetization vector. As illustrated in Fig. 1(a), a current-induced transverse Rashba field should reduce $M_{\mathrm{Z}}$ from its zero-current value of $M_{\mathrm{S}}$, from which the magnitude of $H_{\mathrm{R}}$ can be determined if the out-of-plane anisotropy field $H_{\mathrm{K}}$ is known. This strip exhibited a square hysteresis loop (Fig. 1(b)) with its coercive field $\mu_{0} H_{\text {nuc }} \approx 30 \mathrm{mT}$ governed by reverse domain nucleation. A square field waveform of amplitude $\mu_{0} H_{\text {app }}=36 \mathrm{mT}$ was applied to reverse the strip magnetization alternately, generating a step response in the MOKE signal as shown in Fig. 1(c). The amplitude of the step, proportional to $M_{\mathrm{Z}}$, was measured as a function of injected current density $J$. To attain a sufficient signal-to-noise ratio in the step data, the bandwidth of the MOKE system was limited to $30 \mathrm{kHz}$ and 100 switching cycles were averaged. For each value of injected current density, at least three sets of averaged MOKE step signal were obtained after re-centering the laser spot to gain statistics that accounted for drift in the MOKE signal. Each presented data point is the mean of these multiple measurements, with the corresponding error bar showing the standard deviation. The maximum injected current was $1.6 \mathrm{~mA}$, which corresponds to a current density of $J=8 \times 10^{11} \mathrm{~A} / \mathrm{m}^{2}$ averaged across the combined $\mathrm{Pt}$ and Co cross section, assuming a current distribution in the $\mathrm{Ta} / \mathrm{Pt} / \mathrm{Co}$ stack based on the bulk resistivity of each individual layer.

Fig. 2(a) plots the reduction of $M_{\mathrm{Z}}$ with increasing injected current density. At current density $J=8 \times 10^{11} \mathrm{~A} / \mathrm{m}^{2}, M_{Z}$ reaches $91 \pm 3 \%$ of the zero-current $M_{\mathrm{Z}}$ value. This observed reduction of $M_{\mathrm{Z}}$ could be attributed to two sources: (1) the tilting of the magnetization away from the perpendicular axis due to the transverse Rashba field, and/or (2) the decrease in the saturation magnetization $M_{\mathrm{S}}$ from Joule heating.

To isolate the two sources, we independently quantified both the current-induced Joule heating in the device and the temperature dependence of the saturation magnetization. Measurements of electrical resistance versus injected current at a fixed substrate temperature, and resistance versus substrate temperature at a low sense current, revealed a quadratic increase in 

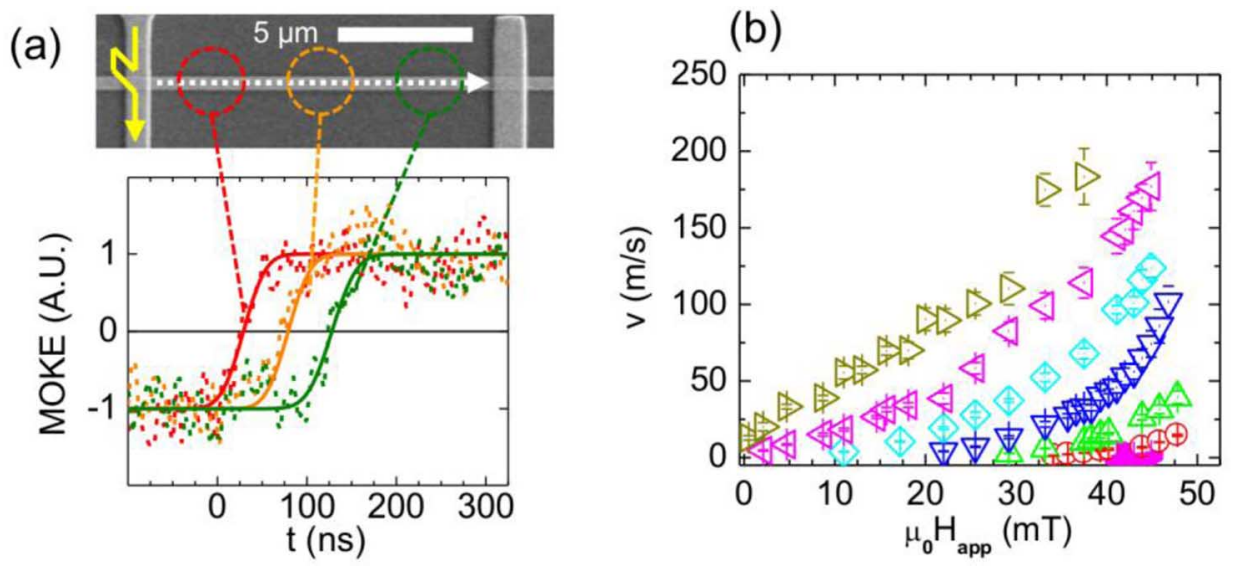

(c)

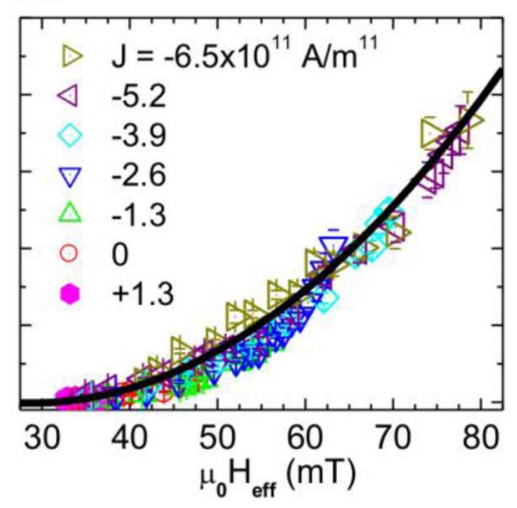

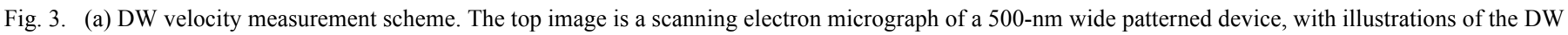

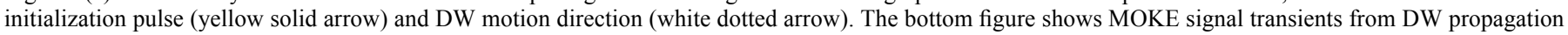

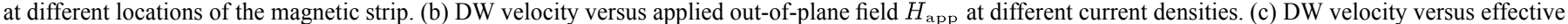
field $H_{\text {eff }}=H_{\text {app }}+\varepsilon J$. The solid curve is a guide for the eye to represent the common dynamic scaling of DW velocities for all driving currents.

the device temperature with current density, $\Delta T=h J^{2}$ with $h=0.84 \pm 0.06 \mathrm{~K} /\left(10^{11} \mathrm{~A} / \mathrm{m}^{2}\right)^{2}$. Using this relation, the decrease in $M_{\mathrm{Z}}$ is re-plotted with respect to the device temperature rise $\Delta T$ in Fig. 2(b). The reduction of $M_{S}$ with increasing substrate temperature from 308 to $373 \mathrm{~K}$, also plotted in Fig. 2(b), was measured with the MOKE technique on a saturated $\mathrm{Pt} / \mathrm{Co} / \mathrm{GdOx}$ continuous film (instead of a patterned device, to eliminate spurious effects from thermal drift).

The decrease of $M_{Z}$ with current closely follows the decrease of $M_{\mathrm{S}}$ with temperature. Therefore, Joule heating alone can completely account for the observed current-induced reduction of $M_{\mathrm{Z}}$ in the $\mathrm{Pt} / \mathrm{Co} / \mathrm{GdOx}$ strip within the measurement uncertainty. We extract an upper limit for the transverse field by taking the lower end of the error bar at $J=8 \times 10^{11} \mathrm{~A} / \mathrm{m}^{2}$ $(\Delta T=55 \mathrm{~K})$, where the normalized $M_{\mathrm{Z}}$ decreases to $88 \%$ of the zero-current $M_{\mathrm{Z}}$ and Joule heating alone reduces $M_{\mathrm{Z}}$ to $92 \%$. In this case, the magnetization tilting angle from the perpendicular axis is $\theta=\arccos (88 / 92)=17^{\circ}$. Neglecting the effect of the applied field $H_{\text {app }}$ that is much smaller than the anisotropy field $H_{\mathrm{K}}$, the transverse Rashba field is $\mu_{0}\left|H_{\mathrm{R}}\right| \approx$ $\mu_{0} H_{\mathrm{K}} \sin \theta=0.23 \mathrm{~T}$ at $J=8 \times 10^{11} \mathrm{~A} / \mathrm{m}^{2}$. Thus, the estimated magnitude of the current-induced Rashba field in $\mathrm{Pt} / \mathrm{Co} / \mathrm{GdOx}$ is $H_{\mathrm{R}}=0 \pm 0.3 \mathrm{~T} / 10^{12} \mathrm{~A} / \mathrm{m}^{2}$, where the large error bar is related to the weak dependence of $M_{Z}$ on tilting angle for small angles. Our measurement is statistically consistent with the values reported by [9]-[11] and is at least a factor of 3 smaller than that reported by [8].

\section{Current-Induced Domain Wall Motion}

The velocities of DWs, driven by combinations of applied out-of-plane field and injected current, were measured using the time-resolved MOKE technique described in detail in [2] and [15]. DWs were initialized by the Oersted field from a 25-ns current pulse $(\sim 100 \mathrm{~mA})$ injected through the $\mathrm{Cu}$ line orthogonal to the $\mathrm{Pt} / \mathrm{Co} / \mathrm{GdOx}$ strip as illustrated in Fig. 3(a). The DW was then driven along the strip by field output by the electromagnet and/or current output by a voltage pulse generator. Here, we summarize key results and analyses of our high-speed DW motion measurements [2] on a similar $\mathrm{Pt} / \mathrm{Co} / \mathrm{GdOx}$ strip to the one in Section III. This strip had a nucleation field of $\mu_{0} H_{\text {nuc }} \approx 50$ $\mathrm{mT}$, which set the maximum applied field for driving a single initialized DW.

DW velocity-versus-field curves at several injected current densities are shown in Fig. 3(b). With increasing current in the same direction as the field-driven DW motion (defined as $J<$ 0 ), the velocity increases. Because the DW velocity increases nonlinearly with driving field (or current) and was found to depend weakly on temperature in separate measurements, DW motion shown in Fig. 3 is still in the thermally activated regime, despite the large velocities exceeding $1 \mathrm{~m} / \mathrm{s}$ and approaching $200 \mathrm{~m} / \mathrm{s}$ in the fastest cases.

A finite-temperature micromagnetics study by Martinez [16] shows that fast $(v>1 \mathrm{~m} / \mathrm{s})$ thermally activated DW propagation in an out-of-plane magnetized nanostrip occurs by DW precession, i.e., continuously transforming between the Bloch and Néel configurations. This occurs because a DW can more readily overcome the pinning potential energy barrier by exploiting both the translational and precessional degrees of freedom. A related study [17] shows that a strong transverse Rashba field (there taken as $1 \mathrm{~T} /\left(10^{12} \mathrm{~A} / \mathrm{m}^{2}\right)$ as suggested by [8]) increases the threshold driving force required for sustained DW motion by suppressing DW precession. Comparing Fig. 5 in [17] and Fig. 9 in [16], the current density above which the DW dynamics escape the creep regime increases by a factor of 4 in the presence of the Rashba field compared to the zero-Rashba field case. With the large transverse field strongly favoring the Bloch configuration while raising the energy penalty for the Néel configuration, the low-energy precessional mode is disabled and the DW can propagate only by rigid translation at higher driving currents. In the thermally-activated regime, this leads to a decrease in the DW velocity at a given driving force.

These computational studies by Martinez [16], [17], though based on conditions quantitatively different from our measurements, indicate that the Rashba effect should have a qualitatively observable influence on current-driven DW dynamics. Specifically, if there was a strong current-induced transverse Rashba field, the velocity of thermally activated 
DW motion at a fixed driving field might decrease abruptly at a large current density corresponding to the onset of rigid translation (stabilization against precession). Such a drop in the DW velocity at high current densities is not apparent in the data of Fig. 3(b), suggesting that the Rashba field is insufficient to prevent precessionally-assisted DW creep motion up to the maximum current densities applied. The lack of a transition in DW dynamics brought on by an in-plane transverse Rashba field is made more evident by plotting the DW velocity against the "effective out-of-plane field" $\mu_{0} H_{\text {eff }}=\mu_{0} H_{\text {app }}+\varepsilon J$, with the spin-torque efficiency $\varepsilon=-6.3 \mathrm{mT} /\left(10^{11} \mathrm{~A} / \mathrm{m}^{2}\right)$ equating the effect of current to an additional out-of-plane field. As shown in Fig. 3(c), all DW velocity data plotted against $H_{\text {eff }}$ converge to a common curve, which indicates that the effect of current can be entirely explained as an effective out-of-plane field without an additional in-plane Rashba field. Joule heating at high currents can account for small deviations from the common curve, as we observed in separate measurements that a temperature rise of $20 \mathrm{~K}$ increased the DW velocity in this regime by as much as a factor of $\sim 2$. This convergence also reveals that even when a DW is driven by a large current and vanishingly small field, its mode of motion is the same as when it is driven by a large field and vanishingly small current. Therefore, DWs in the $\mathrm{Pt} / \mathrm{Co} / \mathrm{GdOx}$ strip move by precession under all driving conditions, and there is no evidence of a Rashba field altering DW dynamics from precession to rigid translation.

\section{CONCLUSION}

The influence of in-plane injected current was investigated in submicrometer-wide strips of $\mathrm{Pt} / \mathrm{Co} / \mathrm{GdOx}$, which was expected to exhibit a large Rashba effect. No strong effective Rashba field was evident in our measurements, despite the highly efficient current-driven DW motion. Although injected current decreased the out-of-plane magnetization component consistent with Rashba field-induced magnetization tilting, the results could be entirely accounted for by Joule heating, with measurement uncertainty yielding an upper limit on the Rashba field of $\sim 0.3 \mathrm{~T} /\left(10^{12} \mathrm{~A} / \mathrm{m}^{2}\right)$. Moreover, high-speed DW velocity measurements did not indicate any appreciable Rashba field large enough to suppress DW precession. Our findings reveal that a strong transverse Rashba field does not explain high spin-torque efficiencies of magnitude well above $1 \mathrm{mT} /\left(10^{11} \mathrm{~A} / \mathrm{m}^{2}\right)$ [1], [2], nor is it even necessarily present, in heavy-metal/ferromagnet/oxide structures. Efficient current-driven DW dynamics must arise from interfacial torques of different symmetry and origin, such as those arising from the spin Hall effect [11], indirectly from the Rashba effect [5]-[7], or from other unexplored phenomena that result in torques allowed by symmetry considerations [7].

\section{ACKNOWLEDGMENT}

This work was supported under NSF-ECCS 1128439. S.E. acknowledges support by the NSF Graduate Research Fellow- ship Program. The authors thank Eduardo Martinez for additional insights into [16] and [17] and David Bono for technical assistance. Sample devices were fabricated using facilities in the MIT Nanostructures Laboratory and the Center for Materials Science and Engineering at MIT.

\section{REFERENCES}

[1] I. M. Miron, T. Moore, H. Szambolics, L. D. Buda-Prejbeanu, S. Auffret, B. Rodmacq, S. Pizzini, J. Vogel, M. Bonfim, A. Schuhl, and G. Gaudin, "Fast current-induced domain-wall motion controlled by the Rashba effect," Nat. Mater., vol. 10, no. 6, pp. 419-423, Jun. 2011.

[2] S. Emori, D. C. Bono, and G. S. D. Beach, "Interfacial current-induced torques in Pt/Co/GdOx," Appl. Phys. Lett., vol. 101, no. 4, p. 042405 , Jul. 2012.

[3] G. S. D. Beach, M. Tsoi, and J. L. Erskine, "Current-induced domain wall motion,” J. Magn. Magn. Mater., vol. 320, no. 7, pp. 1272-1281, Apr. 2008

[4] M. Cormier, A. Mougin, J. Ferré, A. Thiaville, N. Charpentier, F. Piéchon, R. Weil, V. Baltz, and B. Rodmacq, "Effect of electrical current pulses on domain walls in $\mathrm{Pt} / \mathrm{Co} / \mathrm{Pt}$ nanotracks with out-of-plane anisotropy: Spin transfer torque versus Joule heating," Phys. Rev. B, vol. 81, no. 2, p. 024407, Jan. 2010.

[5] A. Manchon and S. Zhang, "Theory of spin torque due to spin-orbit coupling," Phys. Rev. B, vol. 79, no. 9, p. 094422, Mar. 2009.

[6] K.-W. Kim, S.-M. Seo, J. Ryu, K.-J. Lee, and H.-W. Lee, "Magnetization dynamics induced by in-plane currents in ultrathin magnetic nanostructures with Rashba spin-orbit coupling," Phys. Rev. B, vol. 85 , no. 18, p. 180404 , May 2012.

[7] E. van der Bijl and R. A. Duine, "Current-induced torques in textured Rashba ferromagnets," Phys. Rev. B, vol. 86, no. 9, p. 094406, Sep. 2012.

[8] I. M. Miron, G. Gaudin, S. Auffret, B. Rodmacq, A. Schuhl, S. Pizzini, J. Vogel, and P. Gambardella, "Current-driven spin torque induced by the Rashba effect in a ferromagnetic metal layer," Nat. Mater, vol. 9 , no. 3, pp. 230-234, Mar. 2010.

[9] U. H. Pi, K. Won Kim, J. Y. Bae, S. C. Lee, Y. J. Cho, K. S. Kim, and S. Seo, "Tilting of the spin orientation induced by Rashba effect in ferromagnetic metal layer," Appl. Phys. Lett., vol. 97, no. 16, p. 162507 , Oct. 2010

[10] T. Suzuki, S. Fukami, N. Ishiwata, M. Yamanouchi, S. Ikeda, N. Kasai, and H. Ohno, "Current-induced effective field in perpendicularly magnetized $\mathrm{Ta} / \mathrm{CoFeB} / \mathrm{MgO}$ wire," Appl. Phys. Lett., vol. 98, no. 14, p. 142505, Apr. 2011.

[11] L. Liu, O. J. Lee, T. J. Gudmundsen, D. C. Ralph, and R. A. Buhrman, "Current-induced switching of perpendicularly magnetized magnetic layers using spin torque from the spin Hall effect," Phys. Rev. Lett., vol. 109, no. 9, p. 096602, Aug. 2012.

[12] O. Krupin, G. Bihlmayer, K. Starke, S. Gorovikov, J. E. Prieto, K. Döbrich, S. Blügel, and G. Kaindl, "Rashba effect at magnetic metal surfaces," Phys. Rev. B, vol. 71, no. 20, p. 201403, May 2005.

[13] U. Bauer, S. Emori, and G. S. D. Beach, "Electric field control of domain wall propagation in $\mathrm{Pt} / \mathrm{Co} / \mathrm{GdOx}$ films," Appl. Phys. Lett., vol. 100, no. 19, p. 192408, May 2012.

[14] U. Bauer, S. Emori, and G. S. D. Beach, "Voltage-gated modulation of domain wall creep dynamics in an ultrathin metallic ferromagnet," Appl. Phys. Lett., vol. 101, no. 17, p. 172403, Oct. 2012.

[15] S. Emori, D. C. Bono, and G. S. D. Beach, "Time-resolved measurements of field-driven domain wall motion in a submicron strip with perpendicular magnetic anisotropy," J. Appl. Phys., vol. 111, no. 7, p. 07D304, Mar. 2012.

[16] E. Martinez, "The stochastic nature of the domain wall motion along high perpendicular anisotropy strips with surface roughness," J. Phys.: Condens. Matter, vol. 24, no. 2, p. 024206, Jan. 2012.

[17] E. Martinez, "Micromagnetic analysis of the Rashba field on currentinduced domain wall propagation," J. Appl. Phys., vol. 111, no. 3, p. 033901 , Feb. 2012. 